\title{
Spectropolarimetry of CV's; Signatures of Magnetic Fields in Accretion Flows
}

\author{
Danny Steeghs, Keith Horne \\ Physics $\mathcal{E}$ Astronomy, North Haugh, St.Andrews, Fife KY16 9SS, \\ Scotland
}

\begin{abstract}
We present preliminary results of spectropolarimetric observations of several cataclysmic variables using the ISIS spectropolarimeter on the $4.2 \mathrm{~m}$ William Hershell Telescope at La Palma. Our aim is to look for Zeeman signatures of magnetic fields in cataclysmic variables. This would provide a direct measurement of magnetic fields in the accretion flows of CVs. Our current dataset allows us to set upper limits to the presence of magnetic fields and constrain the field geometry in different types of cataclysmic variables.
\end{abstract}

\section{Introduction}

Theoretical models of magnetic turbulence as a possible angular momentum transport mechanism in accretion discs have reached a sophisticated level (e.g. Balbus, this volume). Unfortunately, no measurements of magnetic fields in accretion discs are currently available.

Emission lines formed in a magnetic region will be partially polarised due to Zeeman split energy levels. The splitting is proportional to the local magnetic field strength and the emission components of the Zeeman triplet have different polarisation characteristics. The Stokes components depend on the direction of the magnetic field compared with the line of sight, the central component is linearly polarised, the two shifted components are circular polarised. If one resolves these components and measure their polarisation characteristics, one can infer the magnitude and direction of the magnetic field in the lineforming region. We expect the magnetic field geometry to be quite complicated and velocity broadening will make it impossible to resolve the individual Zeeman components. One can still, however, study the field geometry by looking at the wavelength dependent polarisation of the emission lines, which will be a convolution of the different magnetised emission sites with different field strengths and doppler shifts.

\section{Polarisation signatures}

The fact that the polarisation of the different Zeeman components depends on the direction of the magnetic field allows us to use the binary system rotation to sample the magnetic field geometry. The phase dependent signatures of the magnetic patches in the system (figure 1 , left) reproduce a potentially observable 

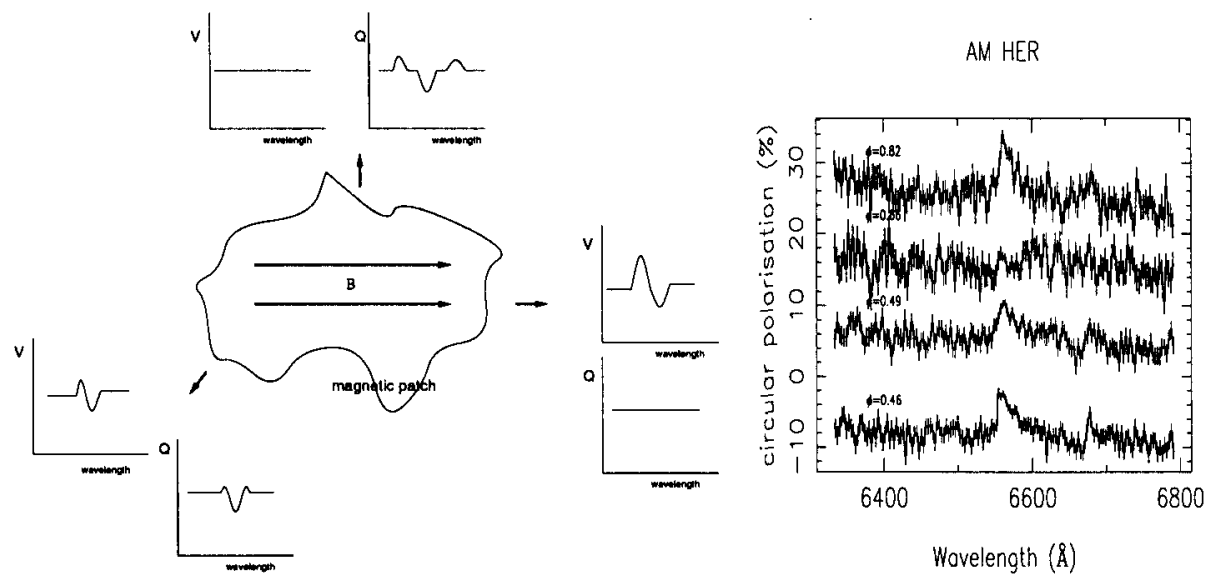

Figure 1. left:Schematic signature of a magnetised patch. Linear (Q) and circular (V) polarisation profiles depend on the angle of the line of sight with the magnetic field direction. right:Observed circular polarisation of AM Her at different binary phases (offset between spectra is $12 \%)$.

line polarisation as a function of orbital phase. This is analogous the use of phase resolved spectroscopy to backproject the observed emission contributions at different velocities into a distribution map, a technique known as Doppler tomography (Horne, this volume).

By simulating the polarisation signatures of theoretical models, for example of magnetised accretion discs, we can look for these signatures to provide observational feedback to these theoretical models.

\section{First data}

We have currently observed a sample of 6 bright CVs using the ISIS spectropolarimeter on the $4.2 \mathrm{~m}$ William Hershell Telescope at La Palma. To illustrate the technique and test the algorithms, the polarisation of a polar, like AM Her, is very helpful. This magnetic CV contains a highly magnetised white dwarf which funnels all the accreting material onto its fieldlines. This is a fairly simple field geometry and more importantly fixed in the binary frame. Figure 1 (right) shows the observed phase dependent spectropolarimetry data, supporting our idea.

We plan to model our current polarisation observations of several CVs to provide upper limits to the magnetic fields in these systems. Only AM Her shows significant polarisation structure which allows a direct determination of the field geometry. We will use this initial set to fine-tune our technique and perform simulations to explore the possibilities of detecting magnetic fields this way with future large scale telescope facilities. 